\title{
Analysis and Evaluation of Dynamic Load Balancing in IEEE 802.11b Wireless Local Area Networks
}

\author{
Farhad Soleimanian Gharehchopogh \\ Department of Computer Engineering, \\ Hacettepe University, Beytepe, \\ Ankara, Turkey
}

\author{
Zeinab Abbasi Khalifehlou \\ Department of Computer Engineering, \\ Shabestar Branch, Islamic Azad University, \\ Shabestar, Iran
}

\begin{abstract}
IEEE 802.11 WLANs (Wi-Fi) standards are widely developed for presenting internet access public space in hot spots, due to their unique features. The features of wireless local area networks (WLANs) are simple installation with low cost, and support high speed data communications. This study is a discussion and evaluation of the techniques in dynamic load balancing in WLANs. Some of the methods consist of cell breathing (CB) techniques for cooperative load balancing, utility based load balancing and others in WLANs. Finally, we highlighted and compared the techniques according to their applications in IEEE 802.11b WLANs. The results indicate that by means of dynamic load balancing algorithms in WLANs, network performance is maximized.
\end{abstract}

\section{Keywords}

Dynamic Load Balancing, Wireless Local Area Networks, Cell Breathing.

\section{INTRODUCTION}

IEEE 802.11x standards define wireless local area networks (WLANs) which present solutions for wireless broadband access. WLANs continue to grow in capability and accessibility, particularly in enterprise networks or cordless access to private home with short range radio and multiple wireless access points (APs). The IEEE $802.11 \mathrm{~b}$ standard physical layer is a backward-compatible extension of the original radio physical layer in the $2.4 \mathrm{GHz}$ band that supports up to $11 \mathrm{Mbps}$ data rates [1]. These networks try to bring the need to apply smart load balancing techniques to improve network performance. These techniques require the provision of radio measurements from physical and medium access layers.

With the 802.11 based WLAN multimedia applications (such as video, audio, real-time voice over IP) providing high quality of Service (QoS) [2]. The standard presents access to the wireless users only according to physical considerations. This can lead to overloaded access point (AP) and considerable degradation of the QoS [2, 3]. Unbalanced load can to make overloading and network congestion [4]. Load balancing in WLANs is the evaluation of associations in mobile stations at time $t_{k}$ dynamically, and then try to detecting optimal set of mobile stations (MS) under each AP focus on estimation of the states of mobile stations at time $t_{k+1}$ [7].

Due to distributed specification of WLANs, distributed algorithms like DSA/DBA [1], DPOP [6] and ADOPT [6] have been proposed for solving distributed constraint optimization (DCOP) issues. Hence, these algorithms are used for graph coloring and meeting scheduling [7]. Among of them, DPOP is a complete algorithm based on dynamic programming and a utility propagation method that extends tree propagation algorithms to work on arbitrary topologies by means of a pseudo tree structure. This paper deals with load balancing approaches in IEEE WLANs. The paper organized as follows: Next section reviewed previous works in this domain. Section 3, is introduction to load balancing in IEEE 802.11 WLANs. In sections 4 and subsections of them, we are described the dynamic load balancing approaches, CB techniques in dynamic load balancing and multi agent pseudotree repair resolutions. Section 5, is evaluation and comparison of the discussed methods and section 6 , is highlighted the conclusions to be the results of this research.

\section{PREVIOUS WORKS}

In recent years, many researches are considered in WLANs domains such as different researches are considered mobile users tend to group in certain area of interest as hot spots for specifying an uneven distribution of traffic load between network APs are detailed in [8, 9]. Tsao and Hsu [10] considered a new novel dynamic load balancing algorithm for a voice over IP (VoIP) over WLAN (VoWLAN) system.

Also, reference [2] proposed one load balancing procedure for overlapping wireless LAN cells with installed agents in each AP broad cast periodically. The results show that this scheme increases the total wireless throughput and decreases the cell delay. Load balancing mechanisms present an element of congestion relief to those APs with a high density of users [7]. Haidar et al. [2] proposed a new load balancing algorithm based on power management of APs to reduce congestion at hot spots in WLANs. Their algorithm obtains the most congested AP, decreases the transmitted power in discrete steps. Then the process continues until the users' assignment which leads to a high balance index is reached. As a result, the algorithm is capable of reducing the overall congestion at hot spots in WLANs and increases the signal to interference radio (SIR) substantially for cases involving rather large WLANs [2] as well as network throughput are increased.

Balachandran et al. [11] evaluate the effectiveness of two algorithms on improving user service rates and network utilization using simulations. The proposed algorithms are explicit channel switching and network directed roaming for providing hot spot congestion relief while maintaining prenegotiated user bandwidth agreements with the network. They improve the degree of load balance in the system by over 30 percent and user bandwidth allocation by up to 52 percent in comparison to existing schemes that offer little or no load balancing.

References [3, 12] are presented networks controlled frameworks in which client stations send the required information to a central unit and access to the load information for each cell. However, Brickley et al. [13] work 
at enhancement and will attempt to improve the quality that a user will receive by balancing the load between various access points while changing the coverage area of each access point, a technique commonly known as cell breathing with the aim of improving the QoS. Reference [14] developed a set of polynomial time algorithms to detecting the optimal beacon power setting which minimize the load of the most congested AP with presenting the network-wide min-max load balancing. They are presented a new load balancing technique by controlling the AP's coverage range.

However, reference [15] present and analyze user behavior and network performance in public area wireless networks. They detected that the load distribution in terms of bandwidth across APs is highly uneven and not very well correlated with the number of users associated with those APs. Bejerano and Han [16] proposed a new load balancing method by controlling the size of WLAN cells. The proposed technique does not any modifications at the user side neither the standard, but it only requires the ability of changing the transmission power of the AP beacon messages dynamically. It is lead to minimizing the load of the most congested APs in polynomial time. Other studies about traffic load and load balancing techniques in WLANs are explained in $[17,18,19$, 20 and 21].

\section{LOAD BALANCING PROBLEM}

Load balancing problem can lead to overloading and network congestion [4]. To deal with this problem has been discussed solutions which are dynamic load balancing with CB techniques, pseudo-tree repair algorithm and etc which are described in more. In order to increasing overall system throughput, to be increasing number of APs in the mobile station which lead to heavy traffic distributing between them. As shown in the follow figure, the unbalanced load problem among of the APs lead to traffic load or overloading in the WLANs.

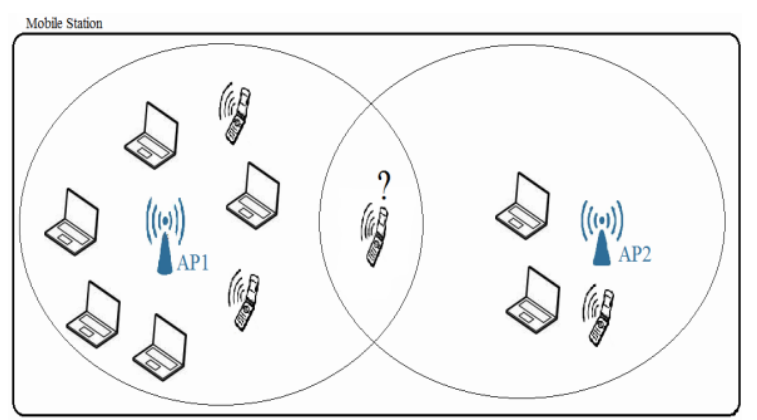

Fig1. Load Balancing Problem in Wireless Stations

Load balancing in IEEE 802.11 WLANs means the number of the active process per AP and a load balancing technique try to create the same number of enable process per cell [22]. Unbalanced problems lead to unacceptable response times and unfair bandwidth assignment and long waiting time in WLANs.

\section{DYNAMIC LOAD BALANCING APPROACH}

In recent years, several dynamic load balancing methods have been proposed for solving this unbalanced loaded problem. Here, some of these points. The heavy traffic of loads is often distributed between the APs unevenly, so it is lead to unavailability of services and unbalanced problem and result overloading by network users. Because of maximizing the network performance, load balancing policy is imperative for distributed of the wireless station to the APs [23].

Each of the wireless station has one or more APs. In dynamic load balancing approach, a wireless station joins to an AP; depend on the number of the available associated stations to the AP and the mean received signal strength indicator (RSSI) [23]. Dynamic load balancing includes three levels which are AP channel auto selection, station join decision level and link observation level [23]. Dynamic load balancing refers to best distribution of the APs to already channels, specifying the behavior that the wireless station choices the AP to connected with it and define link observation when the wireless stations leaves the AP and the roaming function is carried out. In fact, dynamic load balancing differs from traditional approach. In wireless station with some of stations and APs, each station should choice an AP to connect to network.

In dynamic load balancing approach, the average RSSI in WLANs is maximized and both variance of average RSSI value of set stations (VAR) and variance of the number of stations which connected to special AP i.e. VSN are minimized [26]. Common points of all the dynamic load balancing algorithms are dynamic AP selection for connecting to it and capability of station to observing the APs which leaves the wireless station as well as together they have some differences in algorithmic detail for implementing them.

\subsection{Dynamic Load Balancing with Cell Breathing}

$\mathrm{CB}$ is a lateral result in code division multiple access (CDMA) networks [24] lead to reduce the cell coverage when many users are supported. In fact, CB methods modify and repair cell dimensions increasing or decreasing transmitted power typically and try to load balancing improvement among of neighboring APs. CB in IEEE 802.11WLANs alleviates the congestion in host spots. As a result, congested APs decrease the size of their calls and then underutilized APs increase their cells to attract further stations [25]. However, the aim of the transmitted power control is capability for reducing power consumption interference and near far lateral [26].

In this perspective, APs are responsible for computing their own load and let their neighbors identify about it by either periodic or triggered updates [25]. APs are one of the following three states regarding to their load include Fair, Gull and Willing. Fair sate means average load in the neighbor-hoods. In this algorithm APs are able to cooperate for redistributing the load between neighboring cells with standard devices. By means of optimal TPC for data exchange ensures a good utilization of the resources and lead to performance improvement in the networks [24].

\subsection{Dynamic Load Balancing with Multi Agent Pseudo-tree Repair Algorithm}

Decision making is very significant in WLANs management and system capability to reach dynamic changes for quickly move from a previous stable state to a new optimal stable state which between APs is a critical problem. DLB-SDPOP is an extended version of DLB-DPOP algorithm in [27] by incorporating a stabilizing mechanism. DLB-SDPOP algorithm includes three phases which are dept first search (DFS) traversal, self-stabilizing utility propagation and optimal value propagation. In DFS Traversal phase, this algorithm carries out a distributed depth first traversal of the network to establish a pseudo tree structure [27]. Second phase propagates utility messages called UTIL messages that consist of utility vectors sent bottom up along the pseudo tree 
starting from the leaves via tree edges [6]. Optimal value's phases are propagated top down from the root node [28]. In this phase, there are communications between root agents for sending value messages to children agents and so on.

\section{EVALUATION}

IEEE 802.11 standards determine some modulations with distinct physical bit rates such as 1,2 and 11Mbps (megabytes per seconds) for $802.11 \mathrm{~b}$ networks [25]. In the CB discussed techniques, $\mathrm{CB}$ presents a network induced association management without any change in client services. Using TPC for data exchange ensures a better utilization of the resources and so leads to high performance in the WLANs $[24,25]$. It also guarantees an even share of bandwidth between clients as well as a balanced load among of APs. On the other hand, distributed $\mathrm{CB}$ (DCB) and centralized $\mathrm{CB}$ called CCB [24] uses the load metric by keep size of the cells as fixed and exchanging data by means of optimal TPC mechanisms.

In CB based load balancing algorithm, a collision probability defines for each cell is depended on the number of active users in [28]. In Pseudo-tree Repair algorithm, great property of DLB-SDPOP finds initial assignments firstly to reach steady state is possible which possible by means of selfstabilizing mechanisms [1, 6 and 28]. It uses pseudo tree localized repair mechanisms instead the complete pseudo reconstruction as well as modifies and repairs the affected points in the original pseudo tree retaining the topology [1].

Regarding to the results of algorithm, it consumes more messages only than DPOP which basically happen in the VALUE and UTIL initiation processes at step 2 of it that is described in [1] and effectiveness of pseudo tree repairs and communicates filtering in DLB-SDPOP. This algorithm has fast response time and no long waiting time to perturbations in real time communications for IEEE 802.11b WLANs. On the other hand, scalable techniques in step 2 leverages pseudo tree localized repair effectively and applies up to 50 percent agent simultaneously failing in real time WLANs communications.

The complexity of this algorithm is $\mathrm{O}\left(\mathrm{dom}^{\mathrm{w}}\right)$, where $d o m$ is bounds the domain size and $w$ is maximum inducted width of all the localized pseudo trees involved in the perturbations in WLANs scenarios [1]. Therefore, SDPOP [24] are developed as resolution for meeting scheduling problem handles up to 10 percent of the agents having simulations perturbations. Regarding to these, DLB-SDPOP algorithm improves WLANs load balancing performance substantially while CB based methods optimize the fairness and performance level of these networks.

\section{CONCLUSION}

This paper discussed in the field of load balancing problem for providing fair service to users of WLANs and balancing the loads among of APs as well as a comparison and evaluation between the two dynamic load balancing methods. The advantages of WLANs technologies consist of mobile, ubiquitous access to enterprise information technology (IT) systems and etc. Load balancing presents an effective and scalable path of managing the ever increasing traffic of the loads. According to these, the overall system throughputs can increase by means of effective balancing AP traffic loads. Also, by creating dynamic load balancing algorithms, the fairness criteria are achieved with high performance in network services and obviously, the WLAN bandwidth is increased unlike the traditional approach in load balancing.

\section{REFERENCES}

[1] Cheng,S., Raja, A., and Howitt, I., DLB-SDPOP: A Multi agent Pseudo-tree Repair Algorithm for Load Balancing in WLANs, 2010 IEEE/WIC/ACM International Conference on Web Intelligence and Intelligent Agent Technology (WI-IAT) 2 (2010), 311318.

[2] Haidar, M., Rizzo, H. A. and AKL, R., User-Based Channel Assignment Algorithm in a Load-Balanced IEEE 802.11 WLAN, International Journal of Interdisciplinary Telecommunications and Networking 1/2 (2009), 66-81.

[3] Bejerano, Y., Han, J., and Li, E., Fairness and Load Balancing in Wireless LANs Using Association Control, $10^{\text {th }}$ International Conference on Mobile Computing and NETWORKING (2004), 315-329.

[4] Zhang, W. and Xing, Z., Distributed breakout vs. distributed stochastic: A Comparative Evaluation on Scan Scheduling, Proceedings of AAMAS-02 Workshop on Distributed Constraint Reasoning, Bologna, Italy (2002), 192-201

[5] Petcu , A., and Faltings, B., DPOP: A Scalable Method for Multi Agent Constraint Optimization, International Joint Conference on Artificial Intelligence 3669/2005 (2005), 266-271.

[6] Modi, P.J., Shen, W., Tambe, M. and Yokoo, M. ADOPT: Asynchronous Distributed Constraint Optimization with Quality Guarantees, AI Journal 161 (2005), 149-180.

[7] Brickley, O. Rea, S. and Pesch, D., Load Balancing for QoS Optimization in Wireless LANs Utilizing Advanced Cell Breathing Techniques, 61st Vehicular Technology Conference, IEEE 3/2005 (2005), 2101-2252.

[8] Magdalena, B. and Paul, C., Characterizing Mobility and Network Usage in a Corporate Wireless Local-Area Network, Mo-biSys '03 Proceedings of the 1st International Conference on Mobile systems, Applications and Services (2003), 303-316.

[9] Tsao S.L. and Hsu, C.C, A Dynamic Load Balancing Scheme for VoIP over WLANs: Lecture Notes in Computer Science, JOURNAL OF INFORMATION SCIENCE AND ENGINEERING 24 (2008), 47-60.

[10] Velyos, H., Aleo, V. and Karlsson,G., Load Balancing in Overlapping Wireless LAN Cells, IEEE International Conference on Communications, 2004, ICC'04 7 (2004), 3833-3836.

[11] Balachandran, A. Bahl,P. and Voelker, G.M., Hot Spot Congestion Relief in Public Area Wireless Networks, $4^{\text {th }}$ IEEE Workshop on Mobile Computing Systems and Applications (2002), 70-80.

[12] Bianchi, G., Performance Analysis of the IEEE 802.11 Distributed Coordination Function, IEEE Journal on Selected Areas in Commun 18/3 (2000), 535-547.

[13] Brickley,O., Rea,S., and Pesch, D., Load Balancing for QoS Enhancement in IEEE802.11e WLANs using cell breathing techniques, International Conference in IFIP Mobile and Wireless Communication Networks, Marrakech, Morocco 3669/2005 (2005), 367-378. 
[14] Velayos, H., Karlsson, G. and Aleo, V., Cell Breathing Load Balancing in Wireless LANs, IEEE International Conference on Communications 7 (2004), 3833-3836.

[15] Balachandran, A., Voelker, G.M., Bahl, V. and Rangan, P.V., Characterizing User Behavior and Network Performance in a Public Wireless LAN, in Proc. Of ACM SIGMETRICS (2002), 195-205.

[16] Bejerano, Y. and Han, S. J., Cell Breathing Techniques for Load Balancing in Wireless LANs, IEEE Transaction on Mobile Computing 8/6 (2006), 735-751.

[17] Rizzo, H.A, Haidar, M., Akl, R. and Chan, Y., Enhanced Channel, Assignment and Load Distributed in IEEE 802.11 WLANs, IEEE International Conference Signal Processing and Communications (2007), 768-771.

[18] Jabri, I., Divoux, T., Krommenacker, N., and Soudani, A. Cell Breathing Load Balancing in Wireless LANs, IEEE 802.11 Load Balancing: An Approach for QoS Enhancement 15/1 (2008), 16-30.

[19] Wang, Y., Laureie, C., and Ma, A., IEEE 802.11 WLAN Load Balancing Using Adaptive Antennas and Cooperative Controls, IET International Conference on Wireless, Mobile and Multimedia Networks (2006), 1-4.

[20] Y. Bejerano, S. J. Han, and E. Li, Fairness and Load Balancing in Wireless LANs Using Association Control, IEEE/ACM Transaction on Networking 15/3 (2007), $560-571$.

[21] Hamdi,S., Soudani, A., and Rached, T., Experimental Performances Analysis of Load Balancing Algorithms in
IEEE 802.11, International Journal of Computer Science and Information security (IJCSIS) 4/2 (2009), 1-6.

[22] Bejerano,Y. and Han, S., Cell Breathing Techniques for Load Balancing in Wireless LANs, IEEE Transactions on Mobile Computing 6/8 (2009), 725-749.

[23] Sheu, S.T., and Wu, C.C., Dynamic Load Balance Algorithm (DLBA) for IEEE 802.11 Wireless LAN, Journal of Science and Engineering 2/1 (1999), 45-52.

[24] Garcia, E.R. and Paradells, J., Cooperative Load Balancing in IEEE 802.11 Networks with Cell Breathing, IEEE Symposium Computers and Communications,(ISCC 2008) (2008), 1133-1140.

[25] Qiao, D., and Choi, S., New 802.11h Mechanisms Can Reduce Power Consumption, IT Professional 8/2 (2006), 43-48.

[26] Faltings, B., and Petcu, A., S-DPOP: Super Stabilizing, Fault Containing Multi Agent Combinatoria Optimization, IEEE National Conference on Artificial Intelligence (AAAI-05) (2005), 449-454.

[27] Bianchi, G., and Kalman, I.T., Filter Estimation of the Number of Competing Terminals in an IEEE 802.11 Network, 22nd Annual Joint Conference of the IEEE Computer and Communications Societies 2 (2003), 844 852.

[28] Junges, R., and Bazzan, A.L.C., Evaluating the Performance of DCOP Algorithms in a Real World, Dynamic Problem, AAMAS (2) (2008), 599-606. 